\title{
The Representation and Continuity of a Generalized Metric Projection onto a Closed Hyperplane in Banach Spaces
}

\author{
XianFa Luo' and JianYong Wang ${ }^{2}$ \\ ${ }^{1}$ Department of Mathematics, China Jiliang University, Hangzhou 310018, China \\ ${ }^{2}$ Department of Mathematics, Changshu Institute of Technology, Changshu 215500, China
}

Correspondence should be addressed to XianFa Luo; luoxianfaseu@163.com

Received 27 September 2013; Accepted 21 October 2013

Academic Editor: Antonio M. Peralta

Copyright (C) $2013 \mathrm{X}$. Luo and J. Wang. This is an open access article distributed under the Creative Commons Attribution License, which permits unrestricted use, distribution, and reproduction in any medium, provided the original work is properly cited.

Let $C$ be a closed bounded convex subset of a real Banach space $X$ with 0 as its interior and $p_{C}$ the Minkowski functional generated by the set $C$. For a nonempty set $G$ in $X$ and $x \in X, g_{0} \in G$ is called the generalized best approximation to $x$ from $G$ if $p_{C}\left(g_{0}-x\right) \leq$ $p_{C}(g-x)$ for all $g \in G$. In this paper, we will give a distance formula under $p_{C}$ from a point to a closed hyperplane $H\left(x^{*}, \alpha\right)$ in $X$ determined by a nonzero continuous linear functional $x^{*}$ in $X$ and a real number $\alpha$, a representation of the generalized metric projection onto $H\left(x^{*}, \alpha\right)$, and investigate the continuity of this generalized metric projection, extending corresponding results for the case of norm.

\section{Introduction}

Throughout this paper, $(X,\|\cdot\|)$ is a real Banach space with the closed unit ball $B(X)$, and $X^{*}$ is its the topological dual. For a nonempty subset $A$ of $X$, as usual, int $A$ and bd $A$ stand for the interior and the boundary of $A$, respectively. Let $C$ be a bounded closed convex subset of $X$ with $0 \in \operatorname{int} C$. Recall that the Minkowski function $p_{C}: X \rightarrow \mathbb{R}$ with respect to the set $C$ is defined by

$$
p_{C}(x):=\inf \{t>0: x \in t C\}, \quad \forall x \in X .
$$

Let $G$ be a nonempty subset of $X$ and $x \in X$. If there exists $g_{0} \in G$ such that

$$
p_{C}\left(g_{0}-x\right)=\tau_{C}(x, G),
$$

where

$$
\tau_{C}(x, G):=\inf \left\{p_{C}(g-x): g \in G\right\}
$$

is the distance from the point $x$ to the set $G$, then following [1] $g_{0}$ is called the generalized best approximation to $x$ from
$G$. The set of all generalized best approximations to $x$ from $G$ is denoted by $P_{G}^{C}(x)$; that is,

$$
P_{G}^{C}(x)=\left\{g_{0} \in G: p_{C}\left(g_{0}-x\right)=\tau_{C}(x, G)\right\},
$$

which is called the generalized metric projection onto $G$.

When $p_{C}$ is the norm of $X$, the generalized best approximation is reduced to the classical best approximation, which has been studied deeply and extensively since the late 1950s; see [2-4] and references therein. Thus, natural problems are that whether we can extend results in the classical approximation theory to the setting of the generalized approximation. In this direction, some meaning results, such as existences, characterizations, and well-posedness of this kind of approximation, have been established recently; see $[1,5-7]$. In this paper, we will consider the problem of representation of generalized metric projection $P_{H\left(x^{*}, \alpha\right)}^{C}$ onto a closed hyperplane $H\left(x^{*}, \alpha\right)$ and one of continuity of $P_{H\left(x^{*}, \alpha\right)}^{C}$, where $0 \neq x^{*} \in X^{*}, \alpha \in \mathbf{R}$, and

$$
H\left(x^{*}, \alpha\right)=\left\{x \in X: x^{*}(x)=\alpha\right\} .
$$

When $p_{C}$ is the norm of $X$ or, equivalently, $C$ is the closed unit ball $B(X)$ of $X$, this problem has been studied by a 
few authors; see [8-12]. In particular, when $X$ is reflexive, Wang and Yu have given in [10] the representation of $P_{H\left(x^{*}, \alpha\right)}^{B(X)}$, which was further extended by $\mathrm{Ni}$ in [8] to the case of nonreflexive Banach spaces. When $X$ is nearly strictly convex, Wang has shown in [11] that $P_{H\left(x^{*}, \alpha\right)}^{B(X)}$ is norm-to-weak upper semicontinuous on $X$, while, when $X$ is arbitrary Banach space, Zhang and Shi have given in [12] the pointwise continuity of $P_{H\left(x^{*}, \alpha\right)}^{B(X)}$ under an additional condition.

It should be noted that, when one uses a nonnegative convex function $\varphi$ on the Euclidean space $\mathbf{R}^{n}$ satisfying $\varphi(0)=$ 0 and $\varphi(\lambda x)=\lambda \varphi(x)$ for all $x \in \mathbf{R}^{n}$ and $\lambda \geq 0$ as a metric on $\mathbf{R}^{n}$ (i.e., the distance from a point $x$ to a subset $G$ of $\mathbf{R}^{n}$ is defined as $d(x, G)=\inf _{g \in G} \varphi(g-x)$ ), Ferreia and Nemeth have investigated in [13] the problem of the best approximation in $\mathbf{R}^{n}$ and, in particular, given some properties of corresponding metric projections on a hyperplane in $\mathbf{R}^{n}$.

The organization of the present paper is as follows. In Section 2, we define the notions of near strict convexity and weak near strict convexity for the underlying set $C$, which are, respectively, natural extensions of corresponding notions in norm context, and provide an example of a real Banach space $X$ for which $B(X)$ is weakly nearly strictly convex but not nearly strictly convex. In Section 3 , under $p_{C}$, we give a distance formula from a point in $X$ to a hyperplane $H\left(x^{*}, \alpha\right)$ in $X$ and a representation of the generalized metric projection $P_{H\left(x^{*}, \alpha\right)}^{C}$ and consider the continuity of $P_{H\left(x^{*}, \alpha\right)}^{C}$. Results obtained in the present paper extend classical Ascoli Theorem (i.e., the distance formula under the case of norm from a point to a closed hyperplane in a Banach space) and main results in $[8,10-12]$ from the setting of norm to that of the Minkowski functional.

\section{Preliminaries and an Example}

Recall that $(X,\|\cdot\|)$ is a real Banach space with the topological dual $X^{*}, C$ is be a closed bounded convex subset of $X$ with $0 \in \operatorname{int} C$, and $p_{C}$ is the Minkowski function given by (1). Define the polar $C^{\circ}$ of the set $C$ by

$$
C^{\circ}:=\left\{x^{*} \in X^{*}: x^{*}(x) \leq 1, \forall x \in C\right\} .
$$

Then $C^{\circ}$ is a nonempty weakly ${ }^{*}$ compact convex subset of $X^{*}$ with $0 \in \operatorname{int} C^{\circ}$.

We first list some useful properties of the Minkowski function $p_{C}$ which can be proved easily by the definition.

Proposition 1. Let $x, y \in X$ and $x^{*} \in X^{*}$. Then

(i) $p_{C}(x) \geq 0$ and $p_{C}(x)=0 \Leftrightarrow x=0$;

(ii) $x \in C \Leftrightarrow p_{C}(x) \leq 1$ and $x \in$ bd $C \Leftrightarrow \mathrm{p}_{\mathrm{C}}(\mathrm{x})=1$;

(iii) $p(x+y) \leq p(x)+p(y)$ and $p_{C}(t x)=t p_{C}(x)$ for each $t \geq 0$

(iv) $p_{C}(x)=\sup _{y^{*} \in C^{*}} y^{*}(x)$ and $p_{C^{\circ}}\left(x^{*}\right)=\sup _{x \in C^{2}} x^{*}(x)$;

(v) $x^{*}(x) \leq p_{C}(x) p_{C^{\circ}}\left(x^{*}\right)$;

(vi) there exist positive numbers $m_{1}$ and $m_{2}$ such that

$$
m_{1}\|x\| \leq p_{C}(x) \leq m_{2}\|x\| .
$$

We then give the following definitions which will be used in the rest of this paper.

Definition 2. Let $T$ be a set-valued mapping from $X$ into $2^{X}$, where $2^{X}$ is the set of all subsets of $X$.

(i) Let $x \in X$ with $T(x) \neq \emptyset$. Then $T$ is said to be norm-tonorm (resp., norm-to-weak) upper semicontinuous at $x$ if, for each open set (resp., weakly open set) $W \supseteq$ $T(x)$ there exists an open neighborhood $V$ of $x$ such that $T(y) \subseteq W$ whenever $y \in V$.

(ii) $T$ is said to be norm-to-norm (resp., norm-to-weak) upper semicontinuous on $X$ if, for each $x \in X$, $T(x) \neq \emptyset$ and $T$ is norm-to-norm (resp., norm-toweak) upper semicontinuous at $x$.

(iii) $T$ is said to be norm-to-norm continuous on $X$ if, for each $x \in X, T(x)$ is single valued and $T$ is norm-tonorm upper semicontinuous at $x$.

Definition 3. The set $C$ is said to be strictly convex (resp., nearly strictly convex and weakly nearly strictly convex) if each convex subset of bd $C$ is a singleton (resp., relatively compact and relatively weakly compact).

Clearly, the notions of near strict convexity and weak near strict convexity for the set $C$ are extensions of corresponding notions for the unit ball $B(X)$, which were, respectively, posed by Banaś in [14] and by Wang in [11]. In the following we will provide an example to show that the near strict convexity for $B(X)$ is strictly stronger than the weak near strict convexity for $B(X)$.

Example 4. Let $X=l_{2}$ be the space of all square convergent real sequences, endowed with the norm by

$$
\|x\|_{0}=\max \left\{\frac{\|x\|_{2}}{\sqrt{2}},\|x\|_{\infty}\right\}, \quad \forall x \in X,
$$

where $\|\cdot\|_{2}$ and $\|\cdot\|_{\infty}$ are the $l_{2}$-norm and the supremum norm on $X$, respectively. Then $\|\cdot\|_{0}$ is equivalent to the $l_{2}$ norm on $X$ because

$$
\frac{\|x\|_{2}}{\sqrt{2}} \leq\|x\|_{0} \leq\|x\|_{2}, \quad \forall x \in X .
$$

Hence, $\left(X,\|\cdot\|_{0}\right)$ is reflexive. It implies that each convex subset of bd $B(X)$ is relatively weakly compact, and consequently $B(X)$ is weakly nearly strictly convex. Below we show that $B(X)$ is not nearly strictly convex. To this end, let $\left\{e_{n}\right\}$ be the natural basis of $l_{2}$, where the $n$th coordinate of $e_{n}$ is 1 and the other coordinates are 0 . Furthermore, let $x_{1}=e_{1}$ and $x_{n}=e_{1}+e_{n}$ for each $n \geq 2$. We claim that $\operatorname{co}\left\{x_{n}\right\}_{n \geq 1} \subseteq$ bd $B(X)$. Indeed, let $y \in \operatorname{co}\left\{x_{n}\right\}_{n \geq 1}$. Then there exist a positive integer $n$ and a sequence $\left\{\lambda_{i}\right\}_{i=1}^{n}$ with $\left\{\lambda_{i}\right\}_{i=1}^{n} \subseteq[0,1]$ satisfying $\sum_{i=1}^{n} \lambda_{i}=1$ such that $y=\sum_{i=1}^{n} \lambda_{i} x_{i}$. Since $y=e_{1}+\sum_{i=2}^{n} \lambda_{i} e_{i}$, one has that $\|y\|_{\infty}=1$ and

$$
\begin{aligned}
\|y\|_{2} & =\left(1+\sum_{i=2}^{n} \lambda_{i}^{2}\right)^{1 / 2} \leq\left(1+\left(\sum_{i=2}^{n} \lambda_{i}\right)^{2}\right)^{1 / 2} \\
& =\left(1+\left(1-\lambda_{1}\right)^{2}\right)^{1 / 2} \leq \sqrt{2} .
\end{aligned}
$$


It follows that $\|y\|_{0}=1$ and the claim is proved. Since $\left\{x_{n}\right\}_{n=1}^{\infty}$ has no convergent subsequences, one sees that $\operatorname{co}\left\{x_{n}\right\}_{n \geq 1}$ is not relatively compact. This shows that $B(X)$ is not nearly strictly convex.

\section{The Representation and Continuity of Metric Projection onto a Hyperplane}

Let $x \in X$ and define

$$
\begin{gathered}
\sigma(x):=\left\{x^{*} \in X^{*}: x^{*}(x)=p_{C}(x) p_{C^{\circ}}\left(x^{*}\right)\right. \\
\left.=p_{C}(x)^{2}=p_{C^{\circ}}\left(x^{*}\right)^{2}\right\},
\end{gathered}
$$

which is analogous to the dual mapping in Banach spaces. Then, for $x^{*} \in X^{*}$, one obtains from the definition that $\sigma^{-1}\left(x^{*}\right)=\left\{x \in X: x^{*} \in \sigma(x)\right\}$ and

$$
\sigma^{-1}\left(\lambda x^{*}\right)=\lambda \sigma^{-1}\left(x^{*}\right), \quad \forall \lambda \in \mathbf{R} .
$$

Hence, for $0 \neq x^{*} \in X^{*}$ and $x \in X$ (noting that $p_{C^{\circ}}\left(x^{*}\right) \neq 0$ ), one has that

$$
x \in \sigma^{-1}\left(x^{*}\right) \Longleftrightarrow x^{*}(x)=p_{C}(x) p_{C^{\circ}}\left(x^{*}\right)=p_{C^{\circ}}\left(x^{*}\right)^{2}
$$

and so

$$
x \in \sigma^{-1}\left(\frac{x^{*}}{p_{C^{\circ}}\left(x^{*}\right)}\right) \Longleftrightarrow \frac{x^{*}(x)}{p_{C^{\circ}}\left(x^{*}\right)}=p_{C}(x)=1 .
$$

Recall that the hyperplane $H\left(x^{*}, \alpha\right)$ determined by $x^{*} \in$ $X^{*} \backslash\{0\}$ and $\alpha \in \mathbf{R}$ is given by (5) and also that $\tau_{C}\left(x, H\left(x^{*}, \alpha\right)\right)$ is the distance from the point $x$ to $H\left(x^{*}, \alpha\right)$ defined by (3). The following result is an extension of the classical Ascoli Theorem for the distance formula under the case of norm from a pint to a hyperplane in a Banach space; see [3, Lemma 1.2 , p. 24].

Proposition 5. Let $x^{*} \in X^{*} \backslash\{0\}, \alpha \in \mathbf{R}$, and $x \in X$. Then

$$
\tau_{C}\left(x, H\left(x^{*}, \alpha\right)\right)=\frac{\left|\alpha-x^{*}(x)\right|}{p_{C^{\circ}}\left(x^{*}\right)} \operatorname{sign}\left(\alpha-x^{*}(x)\right) .
$$

Proof. Without loss of generality, we assume that $\alpha>x^{*}(x)$. Let $y \in H\left(x^{*}, \alpha\right)$. Then $x^{*}(y)=\alpha$; hence

$$
\alpha-x^{*}(x)=x^{*}(y-x) \leq p_{C}(y-x) p_{C^{\circ}}\left(x^{*}\right)
$$

by Proposition $1(\mathrm{v})$. This implies that $\left(\alpha-x^{*}(x)\right) / p_{\mathrm{C}^{\circ}}\left(x^{*}\right) \leq$ $p_{C}(y-x)$ (noting that $p_{C^{\circ}}\left(x^{*}\right) \neq \emptyset$ ), and therefore

$$
\frac{\alpha-x^{*}(x)}{p_{C^{\circ}}\left(x^{*}\right)} \leq \tau_{C}\left(x, H\left(x^{*}, \alpha\right)\right)
$$

because $y \in H\left(x^{*}, \alpha\right)$ is arbitrary.

To show the converse inequality, let $\epsilon \in\left(0, p_{C^{\circ}}\left(x^{*}\right)\right)$. Then, by Proposition 1(iv), there is $z \in C$ such that

$$
x^{*}(z)>p_{C^{\circ}}\left(x^{*}\right)-\epsilon .
$$

Multiplying two sides of (18) by $\left(\alpha-x^{*}(x)\right) / x^{*}(z)\left(p_{C^{\circ}}\left(x^{*}\right)-\right.$ $\epsilon)$, one has that

$$
\frac{\alpha-x^{*}(x)}{p_{C^{\circ}}\left(x^{*}\right)-\epsilon}>\frac{\alpha-x^{*}(x)}{x^{*}(z)} .
$$

Now let $y=x+\left(\left(\alpha-x^{*}(x)\right) / x^{*}(z)\right) z$. Then, $y \in H\left(x^{*}, \alpha\right)$, and

$$
p_{C}(y-x)=\frac{\alpha-x^{*}(x)}{x^{*}(z)} p_{C}(z) \leq \frac{\alpha-x^{*}(x)}{x^{*}(z)}
$$

because $p_{C}(z) \leq 1$ by Proposition 1(ii) (noting that $z \in C$ ). It follows from (19) and (20) that $\left(\alpha-x^{*}(x)\right) /\left(p_{C^{\circ}}\left(x^{*}\right)-\epsilon\right)>$ $p(y-x)$. Hence, $\left(\alpha-x^{*}(x)\right) /\left(p_{C^{\circ}}\left(x^{*}\right)-\epsilon\right)>\tau_{C}\left(x, H\left(x^{*}, \alpha\right)\right)$. Letting $\epsilon \rightarrow 0$ in this inequality gives

$$
\frac{\alpha-x^{*}(x)}{p_{C^{\circ}}\left(x^{*}\right)} \geq \tau_{C}\left(x, H\left(x^{*}, \alpha\right)\right) .
$$

Thus the converse inequality of (17) follows. The proof is complete.

The first main result of this section is as follows, which gives a presentation of the generalized metric projection onto a closed hyperplane in $X$.

Theorem 6. Let $x^{*} \in X^{*} \backslash\{0\}, \alpha \in \mathbf{R}$, and $x \in X$. Then the following assertion holds:

$$
\begin{aligned}
& P_{H\left(x^{*}, \alpha\right)}^{C}(x) \\
& = \begin{cases}x+\frac{\alpha-x^{*}(x)}{p_{C^{\circ}}\left(x^{*}\right)^{2}} \sigma^{-1}\left(x^{*}\right), & \text { if } \sigma^{-1}\left(x^{*}\right) \neq \emptyset, x^{*}(x)<\alpha, \\
x+\frac{x^{*}(x)-\alpha}{p_{C^{\circ}}\left(x^{*}\right)^{2}} \sigma^{-1}\left(-x^{*}\right), & \text { if } \sigma^{-1}\left(-x^{*}\right) \neq \emptyset, x^{*}(x)>\alpha .\end{cases}
\end{aligned}
$$

Proof. Similar to the proof of Proposition 5, we assume that $\sigma^{-1}\left(x^{*}\right) \neq \emptyset$ and $x^{*}(x)<\alpha$. Let $y \in \sigma^{-1}\left(x^{*}\right)$. Then $x^{*} \in \sigma(y)$; hence

$$
x^{*}(y)=p_{C}(y) p_{C^{\circ}}\left(x^{*}\right)=p_{C}(y)^{2}=p_{C^{\circ}}\left(x^{*}\right)^{2} .
$$

Since $p_{C^{\circ}}\left(x^{*}\right) \neq 0$, one has that

$$
p_{C}(y)=p_{C^{\circ}}\left(x^{*}\right) .
$$

Let $z=x+\left(\left(\alpha-x^{*}(x)\right) / p_{C^{\circ}}\left(x^{*}\right)^{2}\right) y$. We then have from (23) that $z \in H\left(x^{*}, \alpha\right)$ and from (24) and Proposition 5 that

$$
\begin{aligned}
p_{C}(z-x) & =\frac{\alpha-x^{*}(x)}{p_{C^{\circ}}\left(x^{*}\right)^{2}} p_{C}(y) \\
& =\frac{\alpha-x^{*}(x)}{p_{C^{\circ}}\left(x^{*}\right)}=\tau_{C}\left(x, H\left(x^{*}, \alpha\right)\right) .
\end{aligned}
$$

This implies that $z \in P_{H\left(x^{*}, \alpha\right)}^{C}(x)$, and further

$$
P_{H\left(x^{*}, \alpha\right)}^{C}(x) \supseteq x+\frac{\alpha-x^{*}(x)}{p_{C^{\circ}}\left(x^{*}\right)^{2}} \sigma^{-1}\left(x^{*}\right) .
$$


To show the reverse inclusion, let $z \in P_{H\left(x^{*}, \alpha\right)}^{C}(x)$. Then, $z \in H\left(x^{*}, \alpha\right)$, and

$$
p_{C}(z-x)=\tau_{C}\left(x, H\left(x^{*}, \alpha\right)\right)=\frac{\alpha-x^{*}(x)}{p_{C^{0}}\left(x^{*}\right)}
$$

thanks to Proposition 5. Noting that

$$
\frac{\alpha-x^{*}(x)}{p_{C^{\circ}}\left(x^{*}\right)}=\frac{x^{*}(z-x)}{p_{C^{0}}\left(x^{*}\right)} \leq p_{C}(z-x),
$$

we get from (27) that

$$
\alpha-x^{*}(x)=x^{*}(z-x)=p_{C}(z-x) p_{C^{0}}\left(x^{*}\right) .
$$

Now let $y=\left(p_{C^{*}}\left(x^{*}\right)^{2} /\left(\alpha-x^{*}(x)\right)\right)(z-x)$. It follows from (29) that

$$
\begin{aligned}
& x^{*}(y)=\frac{p_{C^{\circ}}\left(x^{*}\right)^{2}}{\alpha-x^{*}(x)} x^{*}(z-x)=p_{C^{\circ}}\left(x^{*}\right)^{2}, \\
& p_{C}(y)=\frac{p_{C^{\circ}}\left(x^{*}\right)^{2}}{\alpha-x^{*}(x)} p_{C}(z-x)=p_{C^{\circ}}\left(x^{*}\right) .
\end{aligned}
$$

Hence,

$$
x^{*}(y)=p_{C}(y) p_{C^{0}}\left(x^{*}\right)=p_{C^{\circ}}\left(x^{*}\right)^{2} .
$$

This means that $y \in \sigma^{-1}\left(x^{*}\right)$ by (13), and so

$$
z=x+\frac{\alpha-x^{*}(x)}{p_{C^{C}}\left(x^{*}\right)^{2}} y \in x+\frac{\alpha-x^{*}(x)}{p_{C^{(}}\left(x^{*}\right)^{2}} \sigma^{-1}\left(x^{*}\right) .
$$

Consequently, $P_{H\left(x^{*}, \alpha\right)}^{\mathrm{C}}(x)$ is contained in the right-hand side of (26). The proof is complete.

The following result gives necessary and sufficient conditions for $P_{H\left(x^{*}, \alpha\right)}^{C}(x) \neq \emptyset$.

Proposition 7. Let $x^{*} \in X^{*} \backslash\{0\}, \alpha \in \mathbf{R}$, and $x \in X$ satisfy that $x^{*}(x)<\alpha\left(\right.$ resp., $\left.x^{*}(x)>\alpha\right)$. Then $P_{H\left(x^{*}, \alpha\right)}^{C}(x) \neq \emptyset$ if and only if $x^{*}\left(\right.$ resp., $\left.-x^{*}\right)$ attains its supremum $p_{C^{*}}\left(x^{*}\right)$ (resp., $\left.p_{C^{0}}\left(-x^{*}\right)\right)$ on bd $C$.

Proof. Let $x^{*}, \alpha$, and $x$ be as in Proposition 7, and let $x^{*}(x)<$ $\alpha$. Suppose that $P_{H\left(x^{*}, \alpha\right)}^{C}(x) \neq \emptyset$. Take $y \in P_{H\left(x^{*}, \alpha\right)}^{C}(x)$. Then $x^{*}(y)=\alpha$ and $p_{C}(y-x)=\tau_{C}\left(x, H\left(x^{*}, \alpha\right)\right)$. It follows from Propositions 5 and $1(\mathrm{v})$ that

$$
p_{C}(y-x)=\frac{\alpha-x^{*}(x)}{p_{C^{0}}\left(x^{*}\right)}=\frac{x^{*}(y-x)}{p_{C^{0}}\left(x^{*}\right)} \leq p_{C}(y-x) .
$$

Hence, $x^{*}$ attains its supremum $p_{C}\left(x^{*}\right)$ at $(y-x) /\left(p_{C}(y-\right.$ $x)) \in \operatorname{bd} C$.

Conversely, suppose that $x^{*}$ attains its supremum $p_{C^{\circ}}\left(x^{*}\right)$ at $x_{0} \in \mathrm{bd} C$. Then $x^{*}\left(x_{0}\right)=p_{C^{\circ}}\left(x^{*}\right)$ and $p_{C}\left(x_{0}\right)=1$; hence,

$$
x^{*}\left(p_{C^{\circ}}\left(x^{*}\right) x_{0}\right)=p_{C^{\circ}}\left(x^{*}\right)^{2}=p_{C}\left(p_{C^{\circ}}\left(x^{*}\right) x_{0}\right) p_{C^{\circ}}\left(x^{*}\right) .
$$

This together with (13) implies that $p_{C^{\circ}}\left(x^{*}\right) x_{0} \in \sigma^{-1}\left(x^{*}\right)$, and therefore $\sigma^{-1}\left(x^{*}\right) \neq \emptyset$. By Theorem 6 , one sees that $P_{H\left(x^{*}, \alpha\right)}^{C}(x) \neq \emptyset$. Similarly, we can prove another assertion for the case of $x^{*}(x)>\alpha$.
The second main result of this section is as follows, which describes the continuity of the generalized metric projection $P_{H\left(x^{*}, \alpha\right)}^{C}$ onto the hyperplane $H\left(x^{*}, \alpha\right)$ under the condition that the set $C$ is weakly nearly strictly convex.

Theorem 8. Let the set $C$ be weakly nearly strictly convex, $x^{*} \in$ $X^{*} \backslash\{0\}$, and $\alpha \in \mathbf{R}$. Then the following assertions hold.

(i) Suppose that $x \in X$ satisfies that $x^{*}(x)<$ $\alpha\left(\right.$ resp., $\left.x^{*}(x)>\alpha\right)$ and that $x^{*}\left(\right.$ resp., $\left.-x^{*}\right)$ attains its supremum on bd $C$; then $P_{H\left(x^{*}, \alpha\right)}^{C}$ is norm-to-weak upper semicontinuous at $x$.

(ii) If $x^{*}$ and $-x^{*}$ attain their supremum on bd $C$, then $P_{H\left(x^{*}, \alpha\right)}^{C}$ is norm-to-weak upper semicontinuous on $X$. Furthermore, $P_{H\left(x^{*}, \alpha\right)}^{C}$ is norm-to-norm upper semicontinuous at each point of $H\left(x^{*}, \alpha\right)$.

Proof. (i) Without loss of generality, we assume that $x^{*} \in X^{*} \backslash$ $\{0\}, x \in X$, and $\alpha \in \mathbf{R}$ satisfy $x^{*}(x)<\alpha$ and that $x^{*}$ attains its supremum on bd $C$. We first show that $\sigma^{-1}\left(x^{*}\right)$ is convex. To do this, let $y_{1}, y_{2} \in \sigma^{-1}\left(x^{*}\right)$ and $\lambda \in[0,1]$. Then we obtain from (13) that

$$
\begin{aligned}
x^{*}\left(y_{1}\right) & =p_{C}\left(y_{1}\right) p_{C^{0}}\left(x^{*}\right)=p_{C^{\circ}}\left(x^{*}\right)^{2} \\
& =p_{C}\left(y_{2}\right) p_{C^{\circ}}\left(x^{*}\right)=x^{*}\left(y_{2}\right) .
\end{aligned}
$$

This, together with Proposition 1(v) and (iii), implies that

$$
\begin{aligned}
p_{C^{0}}\left(x^{*}\right)^{2} & =x^{*}\left(\lambda y_{1}+(1-\lambda) y_{2}\right) \\
& \leq p_{C}\left(\lambda y_{1}+(1-\lambda) y_{2}\right) p_{C^{\circ}}\left(x^{*}\right) \\
& \leq\left(\lambda p_{C}\left(y_{1}\right)+(1-\lambda) p_{C}\left(y_{2}\right)\right) p_{C^{\circ}}\left(x^{*}\right) \\
& =p_{C^{\circ}}\left(x^{*}\right)^{2} .
\end{aligned}
$$

Hence, $\lambda y_{1}+(1-\lambda) y_{2} \in \sigma^{-1}\left(x^{*}\right)$ by (13), and $\sigma^{-1}\left(x^{*}\right)$ is convex.

We then show that $\sigma^{-1}\left(x^{*}\right)$ is weakly compact. Since $\sigma^{-1}\left(x^{*} / p_{C^{0}}\left(x^{*}\right)\right) \subseteq$ bd $C$ by $(14)$ and since $\sigma^{-1}\left(x^{*} / p_{C^{0}}\left(x^{*}\right)\right)=$ $\left(1 / p_{C^{C}}\left(x^{*}\right)\right) \sigma^{-1}\left(x^{*}\right)$ by $(12)$, one sees that $\sigma^{-1}\left(x^{*} / p_{C^{*}}\left(x^{*}\right)\right)$ is a convex subset of bdC. It follows that $\sigma^{-1}\left(x^{*} / p_{C^{\circ}}\left(x^{*}\right)\right)$ is relatively weakly compact because $C$ is weakly nearly strictly convex; hence, $\sigma^{-1}\left(x^{*}\right)$ is relatively weakly compact. Thus, to complete the proof, it suffices to show that $\sigma^{-1}\left(x^{*}\right)$ is weakly closed. To do this, let $\left\{x_{\delta}\right\}$ be a net in $\sigma^{-1}\left(x^{*}\right)$ convergent weakly to some $\bar{x} \in X$. Since

$$
x^{*}\left(x_{\delta}\right)=p_{C}\left(x_{\delta}\right) p_{C^{0}}\left(x^{*}\right)=p_{C^{C}}\left(x^{*}\right)^{2} \quad \forall \delta
$$

and since $p_{C}$ is weakly lower semicontinuous by $[15$, Theorem 2.2.1, page 60], we have that

$$
\begin{aligned}
p_{C^{\circ}}\left(x^{*}\right)^{2} & =x^{*}(\bar{x})=\lim _{\delta} x^{*}\left(x_{\delta}\right)=\lim _{\delta} p_{C}\left(x_{\delta}\right) p_{C^{\circ}}\left(x^{*}\right) \\
& \geq p_{C}(\bar{x}) p_{C^{0}}\left(x^{*}\right) .
\end{aligned}
$$


Noting that $x^{*}(\bar{x}) \leq p_{C}(\bar{x}) p_{C^{\circ}}\left(x^{*}\right)$, we get that

$$
x^{*}(\bar{x})=p_{C}(\bar{x}) p_{C^{0}}\left(x^{*}\right)=p_{C^{\circ}}\left(x^{*}\right)^{2} .
$$

Hence, $\bar{x} \in \sigma^{-1}\left(x^{*}\right)$ by (13), and the weak closedness of $\sigma^{-1}\left(x^{*}\right)$ is proved.

Finally, we show that $P_{H\left(x^{*}, \alpha\right)}^{C}$ is norm-to-weak upper semicontinuous at $x$. Otherwise, there exist a weakly open set

$$
W \supseteq P_{H\left(x^{*}, \alpha\right)}^{C}(x)
$$

and a sequence $\left\{x_{n}\right\} \subseteq X$ with $\left\|x_{n}-x\right\| \rightarrow 0$ such that $P_{H\left(x^{*}, \alpha\right)}^{C}\left(x_{n}\right) \nsubseteq W$. Since $x^{*}(x)<\alpha$ and $\left\|x_{n}-x\right\| \rightarrow 0$, we may assume that each $x^{*}\left(x_{n}\right)<\alpha$. Now take $y_{n} \in P_{H\left(x^{*}, \alpha\right)}^{C}\left(x_{n}\right) \backslash W$ for each $n$. By Theorem 6 , there exists $z_{n} \in \sigma^{-1}\left(x^{*}\right)$ such that $y_{n}=x_{n}+\left(\left(\alpha-x^{*}\left(x_{n}\right)\right) / p_{C^{\circ}}\left(x^{*}\right)\right) z_{n}$ for all $n$. Using the weak compactness of $\sigma^{-1}\left(x^{*}\right)$, one has a subsequence $\left\{z_{n_{k}}\right\}$ of $\left\{z_{n}\right\}$ such that $\lim _{k} z_{n_{k}}=z$ weakly for some $z \in \sigma^{-1}\left(x^{*}\right)$. Therefore,

$$
\text { weak }-\lim _{k} y_{n_{k}}=y:=x+\frac{\alpha-x^{*}(x)}{P_{C^{\circ}}\left(x^{*}\right)} z \in P_{H\left(x^{*}, \alpha\right)}^{C}(x) \text {. }
$$

This and (40) imply that $y \in W$. Since $W$ is weakly open, one has that $y_{n_{k}} \in W$ for sufficiently large $k$, which contradicts the choice of $y_{n_{k}}$ and the proof of assertion (i) is complete.

(ii) Let $x \in H\left(x^{*}, \alpha\right)$. Note that the norm-to-norm upper semicontinuity of $P_{H\left(x^{*}, \alpha\right)}^{C}$ at $x$ implies the norm-to-weak upper semicontinuity of $P_{H\left(x^{*}, \alpha\right)}^{C}$ at $x$. It suffices to verify that $P_{H\left(x^{*}, \alpha\right)}^{C}$ is norm-to-norm upper semicontinuous at $x$. To this end, let $W$ be an open neighborhood of $P_{H\left(x^{*}, \alpha\right)}^{C}(x)=x$. Then there exists a positive number $\delta_{0}$ such that $B\left(x, \delta_{0}\right) \subseteq$ $W$, where $B\left(x, \delta_{0}\right)$ denotes the closed ball with center $x$ and radius $\delta_{0}$. Below we show that there is $\delta \in\left(0, \delta_{0}\right]$ such that $P_{H\left(x^{*}, \alpha\right)}^{C}(y) \subseteq B\left(x, \delta_{0}\right)$ whenever $\|y-x\|<\delta$ or, equivalently (noting that if $\delta \in\left(0, \delta_{0}\right]$, one always has that $P_{H\left(x^{*}, \alpha\right)}^{C}(y)=$ $y \in B\left(x, \delta_{0}\right)$ whenever $y \in H\left(x^{*}, \alpha\right)$ and $\left.y \in B(x, \delta)\right)$,

$$
\frac{\alpha-x^{*}(x)}{p_{C^{\circ}}\left(x^{*}\right)^{2}}\|z\| \leq \delta_{0}
$$

for each $z \in \sigma^{-1}\left(x^{*}\right)$ if $x^{*}(y)<\alpha$,

$$
\frac{x^{*}(x)-\alpha}{p_{C^{\circ}}\left(x^{*}\right)^{2}}\|z\| \leq \delta_{0}
$$

$$
\text { for each } z \in \sigma^{-1}\left(-x^{*}\right) \text { if } x^{*}(y)>\alpha
$$

whenever $\|y-x\|<\delta$ due to Theorem 6 .

To proceed, we first verify

$$
\|z\| \leq \frac{p_{\mathrm{C}^{\circ}}\left(x^{*}\right)}{m_{1}}, \quad \forall z \in \sigma^{-1}\left(x^{*}\right),
$$

where the positive number $m_{1}$ is as in Proposition 1 (vi). In fact, let $z \in \sigma^{-1}\left(x^{*}\right)$. Then $p_{C}(z)=p_{C^{\circ}}\left(x^{*}\right)$ by (13). This, together with Proposition 1(vi), implies that $\|z\| \leq$ $\left(1 / m_{1}\right) p_{C}(z)=\left(p_{C^{\circ}}\left(x^{*}\right) / m_{1}\right)$, and $(44)$ is proved. Next, take

$$
\delta=\min \left\{\frac{m_{1} \delta_{0}}{\left\|x^{*}\right\|} p_{C^{\circ}}\left(x^{*}\right), \frac{m_{1} \delta_{0}}{\left\|x^{*}\right\|} p_{C^{\circ}}\left(-x^{*}\right), \delta_{0}\right\} .
$$

Then when $\|y-x\|<\delta$ and $x^{*}(y)<\alpha$, one has, for each $z \in \sigma^{-1}\left(x^{*}\right)$, that

$$
\begin{aligned}
\frac{\alpha-x^{*}(x)}{p_{C^{\circ}}\left(x^{*}\right)^{2}}\|z\| & =\frac{x^{*}(x-y)}{p_{C^{\circ}}\left(x^{*}\right)^{2}}\|z\| \leq \frac{\left\|x^{*}\right\|\|x-y\|}{p_{C^{\circ}}\left(x^{*}\right)^{2}}\|z\| \\
& <\frac{\left\|x^{*}\right\| \delta}{p_{C^{\circ}}\left(x^{*}\right)^{2}} \frac{p_{C^{\circ}}\left(x^{*}\right)}{m_{1}} \leq \delta_{0}
\end{aligned}
$$
result.

hence, (42) holds. While when $\|y-x\|<\delta$ and $x^{*}(y)>\alpha$, we can similarly show that (43) is true. Thus, the proof of (ii) is complete.

A similar proof to that of Theorem 8 yields the following

Theorem 9. Let the set $C$ be nearly strictly convex, $x^{*} \in X^{*} \backslash$ $\{0\}$, and $\alpha \in \mathbf{R}$. The the following assertions hold.

(i) Suppose that $x \in X$ satisfies that $x^{*}(x)<\alpha$ (resp., $x^{*}(x)>\alpha$ ) and that $x^{*}$ (resp., $-x^{*}$ ) attains its supremum on bd $C$; then $P_{H\left(x^{*}, \alpha\right)}^{C}$ is norm-to-norm upper semicontinuous at $x$.

(ii) If $x^{*}$ and $-x^{*}$ attain their supremum on bd $C$, then $P_{H\left(x^{*}, \alpha\right)}^{C}$ is norm-to-norm upper semicontinuous on $X$.

Theorem 10. Suppose that the set $C$ is strictly convex and that nonzero continuous linear functional $x^{*}$ and $-x^{*}$ attain, respectively, their supremum on bdC. Then $P_{H\left(x^{*}, \alpha\right)}^{C}$ is normto-norm continuous on $X$.

Proof. Let $y^{*} \in X^{*} \backslash\{0\}$. We assert that $\sigma^{-1}\left(y^{*}\right)$ contains at most one point under the hypothesis made upon the set $C$. To do this, let $y_{1}, y_{2} \in \sigma^{-1}\left(y^{*}\right)$. Then, from the proof of Theorem $8(\mathrm{i})$, one has that

$$
\begin{aligned}
& p_{C}\left(\lambda y_{1}+(1-\lambda) y_{2}\right) \\
& \quad=p_{C}\left(y_{1}\right)=p_{C}\left(y_{2}\right)=p_{C^{\circ}}\left(y^{*}\right), \quad \forall \lambda \in[0,1] ;
\end{aligned}
$$

hence, $\left[y_{1} / p_{C^{\circ}}\left(y^{*}\right), y_{2} / p_{C^{\circ}}\left(y^{*}\right)\right] \quad \subseteq \quad$ bd $C$ by Proposition 1(ii). It follows from the strict convexity of $C$ that $y_{1} / p_{C^{\circ}}\left(y^{*}\right)=y_{2} / p_{C^{\circ}}\left(y^{*}\right)$, that is, $y_{1}=y_{2}$, and that the assertion is proved. Applying this conclusion to $x^{*}$ and $-x^{*}$ (noting that $\sigma^{-1}\left(x^{*}\right)$ and $\sigma^{-1}\left(-x^{*}\right)$ are nonempty by (13) because $x^{*}$ and $-x^{*}$ attain their supremum on bdC), one sees that both $\sigma^{-1}\left(x^{*}\right)$ and $\sigma^{-1}\left(-x^{*}\right)$ are a singleton. Therefore, $P_{H\left(x^{*}, \alpha\right)}^{C}(x)$ is single valued for each $x \in X$ by Theorem 6, and $P_{H\left(x^{*}, \alpha\right)}^{C}$ is norm-to-norm continuous on $X$ by Theorem 9 , which completes the proof of Theorem 9 .

Theorem 11. Suppose that $X$ is reflexive and that the set $C$ is strictly convex. Then $P_{H\left(x^{*}, \alpha\right)}^{C}$ is norm-to-norm continuous on $X$ for each $x^{*} \in X^{*} \backslash\{0\}$.

Proof. Let $x^{*} \in X^{*} \backslash\{0\}$ be arbitrary. Below we will show that $x^{*}$ attains its supremum on bdC. Granting this, the conclusion follows from Theorem 10. To this end, we take a sequence $\left\{x_{n}\right\} \subseteq C$ such that $\lim _{n} x^{*}\left(x_{n}\right)=p_{C^{\circ}}\left(x^{*}\right)$. Since 
the set $C$ is weakly compact (noting that $X$ is reflexive), there exists a subsequence of $\left\{x_{n}\right\}$, denoted still by $\left\{x_{n}\right\}$, such that $\lim _{n} x_{n}=x$ weakly for some $x \in C$. Thus,

$$
p_{C^{\circ}}\left(x^{*}\right)=x^{*}(x) \leq p_{C}(x) p_{C^{\circ}}\left(x^{*}\right) \leq p_{C^{\circ}}\left(x^{*}\right) .
$$

Consequently, $x \in$ bd $C$ because $p_{C}(x)=1$, and $x^{*}$ attains its supremum at $x$. The proof is complete.

\section{Conflict of Interests}

The authors declare that there is no conflict of interests regarding the publication of this paper.

\section{Acknowledgments}

The first author was supported in part by the NNSF of China (Grant no. 11271342) and the NSF of Zhejiang Province (Grant no. LY12A01029).

\section{References}

[1] F. S. De Blasi and J. Myjak, "On a generalized best approximation problem," Journal of Approximation Theory, vol. 94, no. 1, pp. 54-72, 1998.

[2] D. Braess, Nonlinear Approximation Theory, Springer, New York, NY, USA, 1986.

[3] I. Singer, Best Approximation in Normed Linear Spaces by Elements of Linear Subspaces, Springer, New York, NY, USA, 1970.

[4] S. Y. Xu, C. Li, and W. S. Yang, Nonlinear Approximation Theory in Banach Space, Science Press, Beijing, China, 1997, Chinese.

[5] C. Li, "On well posed generalized best approximation problems," Journal of Approximation Theory, vol. 107, no. 1, pp. 96$108,2000$.

[6] C. Li and R. Ni, "Derivatives of generalized distance functions and existence of generalized nearest points," Journal of Approximation Theory, vol. 115, no. 1, pp. 44-55, 2002.

[7] X.-F. Luo, C. Li, and J.-C. Yao, "Anisotropic best -approximation in normed spaces,” Optimization, vol. 60, no. 6, pp. 725-738, 2011.

[8] R. X. Ni, "The representative of metric projection on the linear manifold in Banach spaces," Journal of Mathematical Research and Exposition, vol. 25, no. 1, pp. 99-103, 2005 (Chinese).

[9] H. W. Pang and Suyalatu, "The continuoity of th emetric projections on a closedhyperplane in Banach space," Acta Mathematical Scientia A, vol. 30, no. 4, pp. 1138-1143, 2010 (Chinese).

[10] Y. W. Wang and J. F. Yu, "The character and representation of a class of metric projection in Banach space," Acta Mathematica Scientia A, vol. 21, no. 1, pp. 29-35, 2001 (Chinese).

[11] J. H. Wang, "Metric projections in nonreflexive Banach spaces," Acta Mathematica Scientia A, vol. 26, no. 6, pp. 840-846, 2006 (Chinese).

[12] Z. Zhang and Z. Shi, "Convexities and approximative compactness and continuity of metric projection in Banach spaces," Journal of Approximation Theory, vol. 161, no. 2, pp. 802-812, 2009.

[13] O. P. Ferreira and S. Z. Németh, "Generalized projections onto convex sets," Journal of Global Optimization, vol. 52, no. 4, pp. 831-842, 2012.
[14] J. Banaś, "On drop property and nearly uniformly smooth Banach spaces," Nonlinear Analysis: Theory, Methods \& Applications, vol. 14, no. 11, pp. 927-933, 1990.

[15] C. Zălinescu, Convex Analysis in General Vector Spaces, World Scientific, Singapore, 2002. 


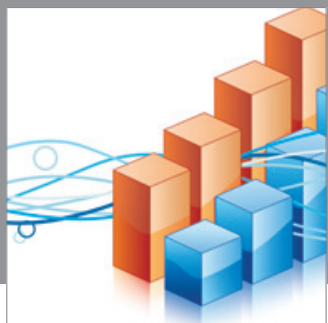

Advances in

Operations Research

mansans

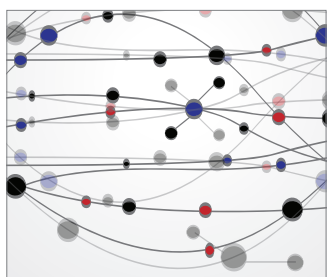

The Scientific World Journal
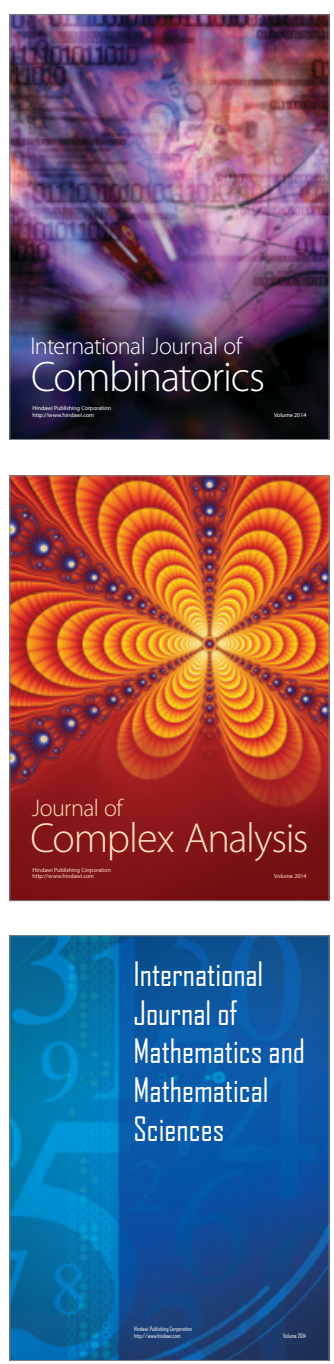
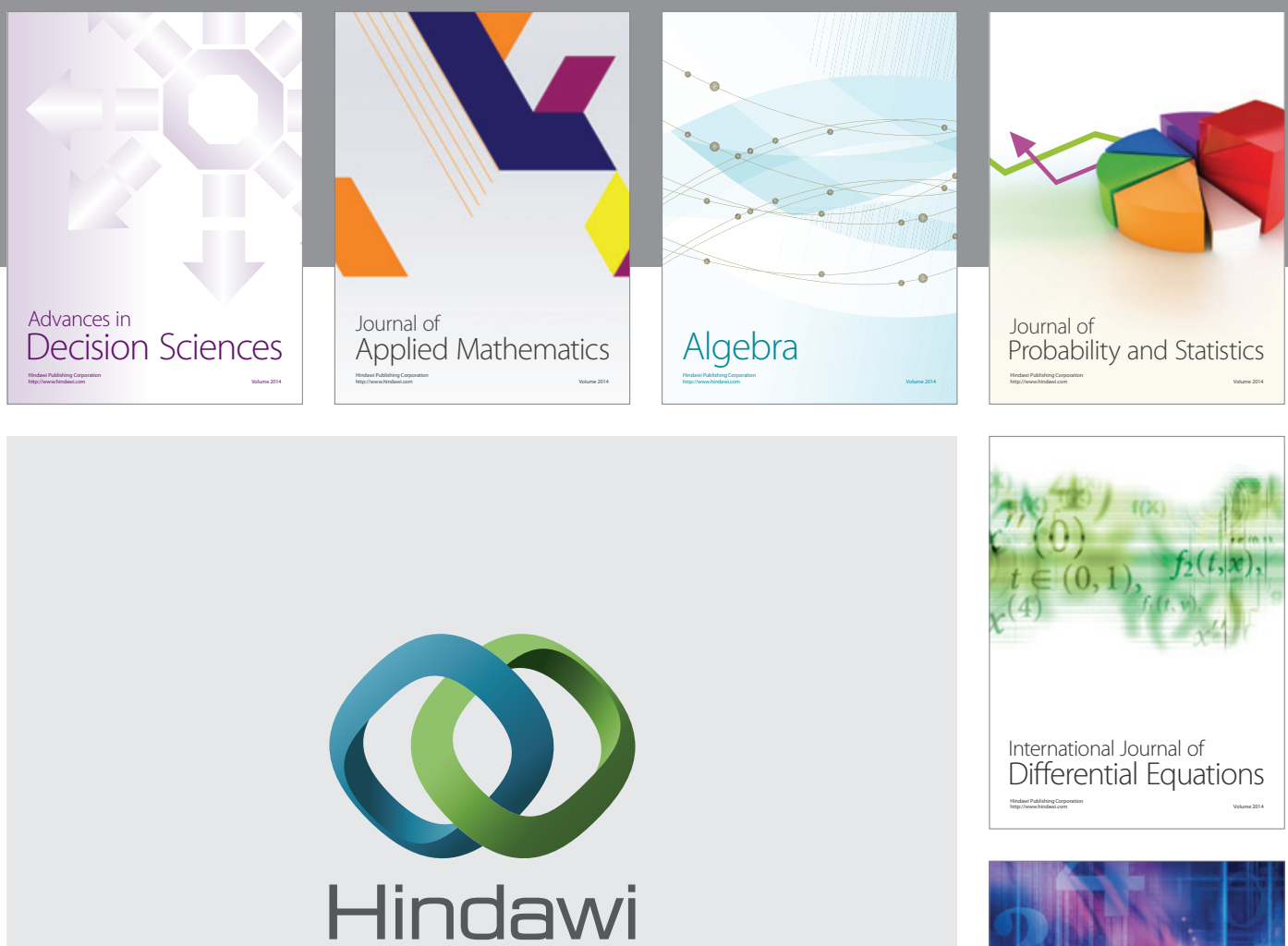

Submit your manuscripts at http://www.hindawi.com
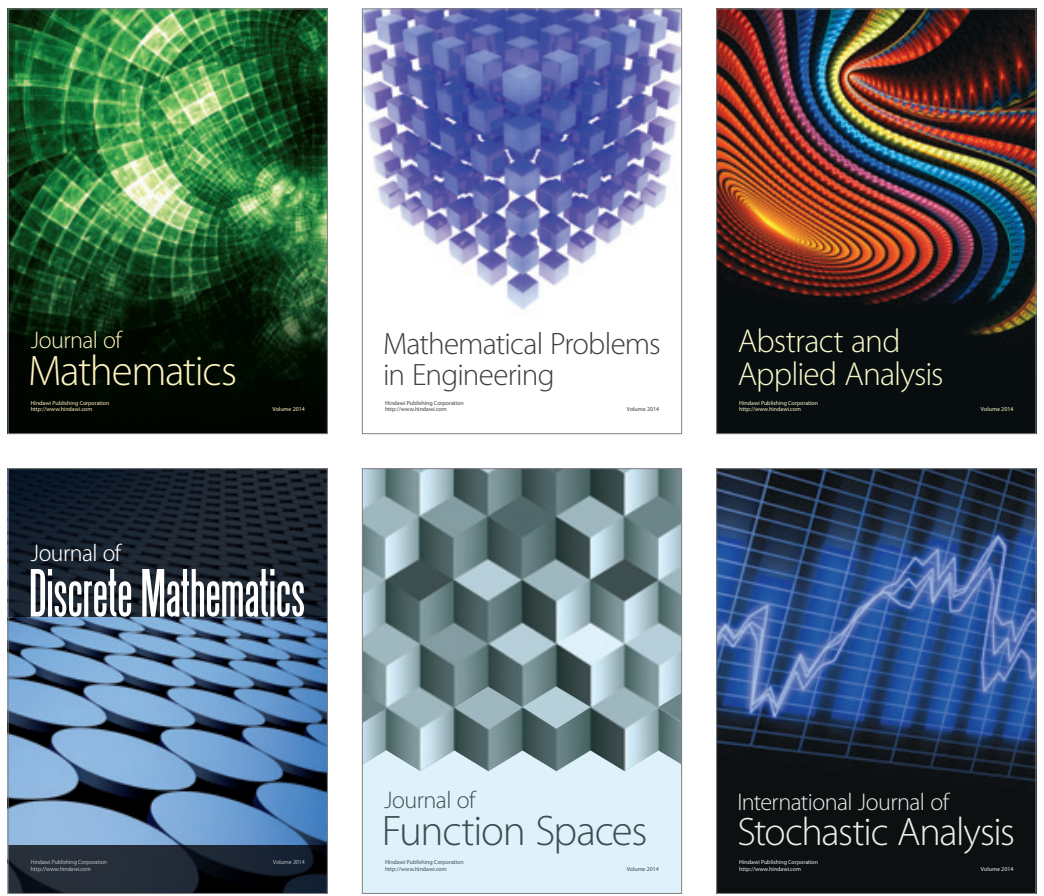

Journal of

Function Spaces

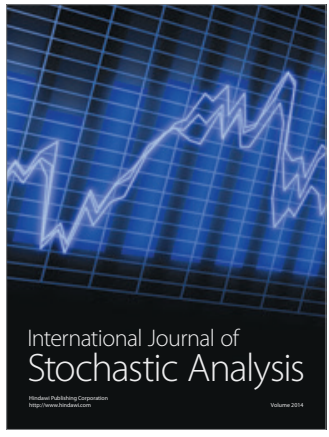

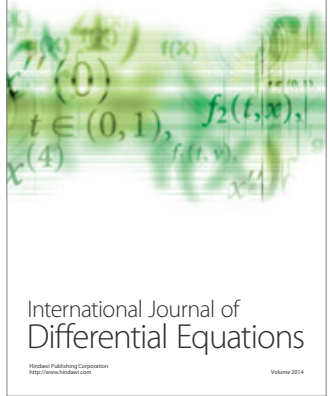
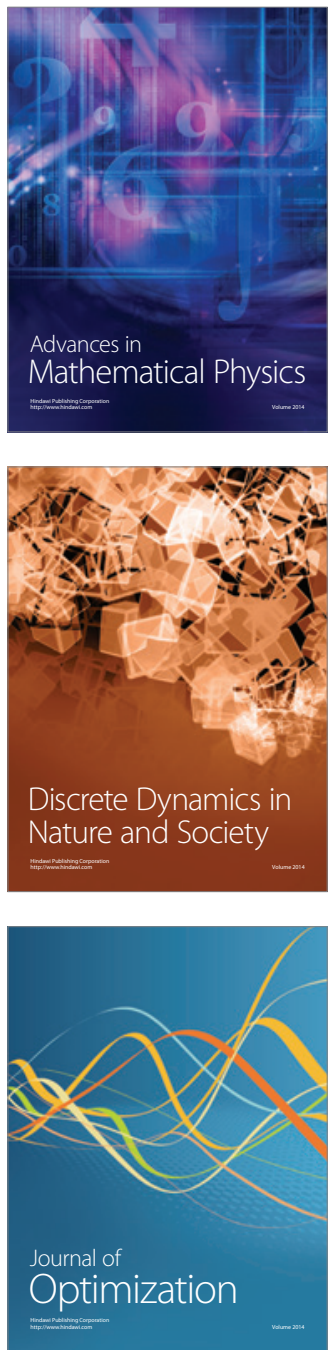